\title{
The Form Birefringence of Macromolecules
}

\author{
By W. L. Bragg and A. B. Pippard \\ Cavendish Laboratory, Cambridge, England
}

(Received 12 December 1952 and in revised form 8 July 1953)

\begin{abstract}
Perutz has shown that the birefringence changes when the water in a haemoglobin crystal is replaced by salt solution. The effect of this substitution in the birefringence is calculated and compared with Perutz's observations. There is agreement as to the order of magnitude of the effect, but the optical data indicated a more elongated molecule $(a / b \sim 1 \cdot 45)$ than that deduced from the $\mathrm{X}$-ray data $(a / b \sim 1 \cdot 3)$. This discrepancy is not to be stressed, since the form of the molecule is not yet certain and the measurements of change in birefringence are very approximate. A further exploration of the effect may yield a useful method of estimating the form of other protein molecules.
\end{abstract}

1

In the preceding paper Perutz (1953) has measured the change in birefringence which occurs when a salt solution replaces water between the molecules of the haemoglobin crystal. The observed birefringence is considered to be due partly to an intrinsic birefringence of the molecule itself, and partly to the elongated form of large molecules of high refractive index which are immersed in a liquid of lower refractive index. Calculations of the form birefringence, and of its variation when the refractive index of the liquid is varied, are made in this note and compared with Perutz's observations.

If parallel spheroids with major and minor axes $a$ and $b$ and dielectric constant $\varepsilon_{2}$ are arranged in a regular way in a liquid of somewhat lower dielectric constant $\varepsilon_{1}$, they are polarized to a greater extent when the electric field is parallel to the long axis than when it is parallel to a short axis. The appropriate equations are derived in $\S 2$ of this note, where it is shown that

$$
\varepsilon=\varepsilon_{1}+\frac{f\left(\varepsilon_{2}-\varepsilon_{1}\right)}{1+(1-f)\left\{\left(\varepsilon_{2}-\varepsilon_{1}\right) / \varepsilon_{1}\right\} L},
$$

$\varepsilon$ being the mean dielectric constant, $f$ the fraction of the volume occupied by the spheroids, and $L$ a depolarizing coefficient depending on the ratio $a / b . L_{\alpha}$, the depolarizing coefficient for an electric field parallel to $a$, is less than $L_{\beta}$ and $L_{\gamma}$ when the spheroid is prolate. By substituting the values of $L$ in the formula, and putting $n_{\alpha}^{2}=\varepsilon_{\alpha}, n_{\beta}^{2}=\varepsilon_{\beta}$, we can evaluate the form birefringence $\left(n_{\beta}-n_{\alpha}\right)$ or $\left(n_{\gamma}-n_{\alpha}\right)$, which of course in this case is of the positive type.

The observed birefringence is the resultant of the intrinsic birefringence and the form birefringence, and we cannot compare the calculated form birefringence with observation because the intrinsic birefringence is not known. We can, however, calculate the change in form birefringence $\Delta\left(n_{\beta}-n_{\alpha}\right)$ when the refractive index of the liquid is varied, and compare this with Perutz's

\footnotetext{
A C 6
}

observations. The form birefringence diminishes when salt solution is substituted for water because $n_{1}$, the refractive index of the liquid, approaches $n_{2}$, the refractive index of the protein. It should vanish when $n_{1}$ is equal to $n_{2}$. The intrinsic birefringence remains unchanged.

We have assumed a value 1.60 for $n_{2}$, the refractive index of protein, based on measurements by Adair \& Adair (1934) of the refractive index of protein solutions. This value may be somewhat uncertain, but a test shows that the calculated values of $\Delta\left(n_{\beta}-n_{\alpha}\right)$ are affected only to a slight extent by comparatively large changes in the value assumed for $n_{2}$. For instance if $n_{2}$ is in error by 0.05 , the calculated value of $\Delta\left(n_{\beta}-n_{\alpha}\right)$ would be changed by only $5 \%$, which is much less than the error of the observations. A complication arises because the protein molecule is believed to be surrounded by a water layer into which the salt does not enter; the effect of this water layer is considered in the next section where it is shown that as a consequence of its presence the 'matching' refractive index is 1.53 and not 1.60 .

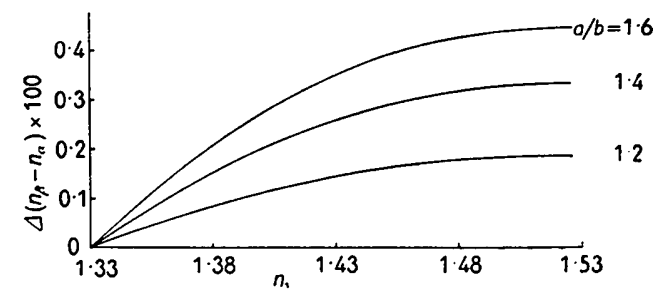

Fig. 1. Calculated variation of $\Delta\left(n_{\beta}-n_{\alpha}\right)$ with $n_{1}$ for $a / b$ ratios of $1 \cdot 2,1 \cdot 4,1 \cdot 6$.

The calculated variation of form birefringence with refractive index is shown in Fig. 1 for axial ratios of $1 \cdot 6,1 \cdot 4,1 \cdot 2$. The experimental measurements of Perutz give the variation for a change of refractive index from 1.370 to 1.397 . The measured change $\Delta\left(n_{\gamma}-n_{\alpha}\right)=\Delta\left(n_{\beta}-n_{\alpha}\right)$ is $0.0008 \pm 0.0002$. This value indicates an axial ratio between $1 \cdot 3$ and $1 \cdot 6$. The 
axial ratio for the haemoglobin molecule deduced from packing considerations (Bragg \& Perutz, 1952a,b) is 1.3 , and the agreement is as satisfactory as can be expected in view of the difficulty of measuring the change in birefringence accurately and the uncertainty as to the precise form of the molecule. In principle, therefore, measurements of form birefringence seem to afford a useful method of studying molecular shape in protein crystals.

\section{2}

(a) We are concerned here to derive an expression for the mean refractive index, and its anisotropy, of an array of similarly oriented transparent ellipsoids, of refractive index $n_{2}$, embedded in a medium of refractive index $n_{1}$, the ellipsoids occupying a fraction $f$ of the total volume. This problem, as was kindly pointed out to us by Dr M. F. Perutz, has already been tackled by Wiener (1912), using a method similar to that which will be used below; but in view of the great length of the paper in which his result is contained, and the unfamiliarity of his notation, his work has remained largely unknown. It seems therefore to be of value to summarize the steps by which the result may be obtained, and to discuss its applicability to the present problem.

(b) First we consider a single ellipsoid of dielectric constant $\varepsilon_{2}\left(=n_{2}^{2}\right)$ immersed in an infinite medium of dielectric constant $\varepsilon_{1}\left(=n_{1}^{2}\right)$. If a uniform electric field, $F$, be applied parallel to one of the principal axes of the ellipsoid, the field within the ellipsoid, $E_{i}$, may be shown (Stratton, 1941, p. 213) to be uniform, and given by the expression

$$
E_{i}=\frac{F}{1+\left\{\left(\varepsilon_{2}-\varepsilon_{1}\right) / \varepsilon_{1}\right\} L},
$$

where $L$, the depolarizing coefficient, is a geometrical constant depending on the ratios of the axes of the ellipsoid. Values of $L$ have been tabulated by Stoner (1945); a few values are given in Table 1 for an ellipsoid of revolution having $F$ parallel and perpendicular to the axis of revolution, $a / b$ being the ratio of the polar to the equatorial axis. Since for any ellipsoid the sum of the three principal values of $L$ is unity, we have that $L_{\|}+2 L_{\perp}=1$.

\section{Table 1}

\begin{tabular}{|c|c|c|c|}
\hline & $a / b$ & $L_{\|}$ & $L_{\perp}$ \\
\hline \multirow[t]{2}{*}{ Plane } & 0 & 1 & 0 \\
\hline & 0.7 & 0.432 & $0 \cdot 284$ \\
\hline \multirow{2}{*}{ Oblate spheroids } & $0 \cdot 8$ & 0.394 & $0 \cdot 303$ \\
\hline & 0.9 & 0.362 & 0.319 \\
\hline \multirow[t]{2}{*}{ Sphere } & $1 \cdot 0$ & $0 \cdot 333$ & $0 \cdot 333$ \\
\hline & $1 \cdot 1$ & 0.308 & $0 \cdot 346$ \\
\hline \multirow{3}{*}{ Prolate spheroids } & $1 \cdot 2$ & 0.286 & 0.357 \\
\hline & $1 \cdot 3$ & 0.266 & $0 \cdot 367$ \\
\hline & $1 \cdot 4$ & $0 \cdot 249$ & 0.376 \\
\hline Cylinder & $\infty$ & 0 & 0.500 \\
\hline
\end{tabular}

It may also be shown that the presence of the ellipsoid is equivalent to the addition of a dipole of moment

$$
\frac{V}{4 \pi} \cdot \frac{\left(\varepsilon_{2}-\varepsilon_{1}\right) F}{1+\left\{\left(\varepsilon_{2}-\varepsilon_{1}\right) / \varepsilon_{1}\right\} L},
$$

$V$ being the volume of the ellipsoid,

from which it follows that if the medium contains a few such ellipsoids, sufficiently far apart for their mutual interactions to be negligible so that $F$ may be put equal to the external applied field, $E_{0}$, then the mean dielectric constant of the mixture is given by the expression

$$
\varepsilon=\varepsilon_{1}+\frac{f\left(\varepsilon_{2}-\varepsilon_{1}\right)}{1+\left\{\left(\varepsilon_{2}-\varepsilon_{1}\right) / \varepsilon_{1}\right\} L},
$$

where $f$ is the volume fraction occupied by the ellipsoids.

(c) Now for an assembly of fairly closely packed ellipsoids it is not permissible to regard $F$, the average field acting on an ellipsoid, as the same as the applied field $E_{0}$, and we must consider in this section the correction needed, which is equivalent to the well known Lorentz correction. For this purpose we consider a fairly sparse random array of oriented ellipsoids. It seems reasonable to suppose that the addition of one more ellipsoid at random to this array will not destroy its random character, and if we consider all the positions which the extra ellipsoid can occupy we conclude that these may include the whole space not already occupied by other ellipsoids. Consequently we may say that the average field acting on an ellipsoid in a not too dense random array is the average field in the medium surrounding the ellipsoids. Denoting this by $F$, we have from (1) an expression for the average field, $E_{i}$, within the ellipsoids. But the applied field, $E_{0}$, must be the same as the average field over the whole space inside and outside the ellipsoids; that is

$$
E_{0}=f E_{i}+(1-f) F .
$$

Hence we may express $E_{i}$ and $F$ in terms of $E_{0}$, and thus calculate the mean polarisation and the mean dielectric constant:

$$
\varepsilon=\varepsilon_{1}+\frac{f\left(\varepsilon_{2}-\varepsilon_{1}\right)}{1+(1-f)\left\{\left(\varepsilon_{2}-\varepsilon_{1}\right) / \varepsilon_{1}\right\} L},
$$

This expression differs from (2) only by the factor $(1-f)$ appearing in the denominator; thus (2) and (3) become identical as the concentration of ellipsoids is made small.

Although (3) has been derived by consideration of a sparse distribution, it appears to be a good approximation even for dense arrays. To take two special cases, for a dielectric sandwich, laminated in planes normal to $E_{0}, L=1$, and (3) becomes

$$
\frac{1}{\varepsilon}=\frac{f}{\varepsilon_{2}}+\frac{1-f}{\varepsilon_{1}}
$$


which is correct for all $f$; while for an array of rods parallel to $E_{0}, L=0$ and

$$
\varepsilon=f \varepsilon_{2}+(1-f) \varepsilon_{1},
$$

which again is correct for all $f$. Probably, indeed, (3) is a good approximation for all $f$ if the ratio $\varepsilon_{2} / \varepsilon_{1}$ is not very great, and particularly good if the unit cell occupied by each ellipsoid has axial ratios comparable to those of the ellipsoid. Thus Rayleigh (1892) has shown that the Lorentz correction (to which (3) reduces when $L=\frac{1}{3}$ ) applies extremely well in a cubic array of spheres, even when the packing is quite close.

(d) Equation (3) may be applied immediately to derive an expression for the birefringence of an array of spheroids, such as a protein crystal. Since we are concerned here only with the component of birefringence due to the shape of the protein molecule we may take $\varepsilon_{2}$ to be isotropic. Then from (3) it immediately follows that

$$
n_{\alpha}^{2}-n_{\beta}^{2}=f\left(n_{2}^{2}-n_{1}^{2}\right)\left(\frac{1}{1+k L_{\alpha}}-\frac{1}{1+k L_{\beta}}\right),
$$

in which $n_{\alpha}$ and $n_{\beta}$ are refractive indices, $L_{\alpha}$ and $L_{\beta}$ depolarizing coefficients, for the electric field lying along the $\alpha$ and $\beta$ directions respectively, and $k$ is written for $(1-f)\left(n_{2}^{2} / n_{1}^{2}-1\right)$, a constant of the crystal. Now for many applications of (4) the molecule is nearly spherical, so that $L_{\alpha}$ and $L_{\beta}$ are near $\frac{1}{3}$. If $L$ is written as $\left(\frac{1}{3}+\gamma\right)$, each term in the second bracket of (4) may be expanded by the binomial theorem with sufficient accuracy for most purposes in the form

so that

$$
\frac{1}{1+\overline{k L}} \fallingdotseq\left(1-\frac{k \gamma}{1+\frac{1}{3} k}\right) /\left(1+\frac{1}{3} k\right)
$$

$$
n_{\beta}^{2}-n_{\alpha}^{2} \fallingdotseq f\left(n_{2}^{2}-n_{1}^{2}\right) \frac{9 k}{(3+k)^{2}}\left(L_{\alpha}-L_{\beta}\right) .
$$

And if, in addition, $f$ is around $\frac{1}{2},\left(n_{\alpha}+n_{\beta}\right)$ is very nearly the same as $\left(n_{2}+n_{1}\right)$ so that

$$
n_{\alpha}-n_{\beta} \fallingdotseq f\left(n_{2}-n_{1}\right) \frac{9 k}{(3+k)^{2}}\left(L_{\beta}-L_{\alpha}\right) \text {. }
$$

It is this expression which is used above in discussing the form birefringence of crystalline haemoglobin.

(e) Finally we must consider how to take account of the fact that a crystalline protein when immersed in a salt solution takes up salt in the interstitial water with the exception of a thin layer immediately surrounding each molecule. The system under consideration is thus a composite system, made up of ellipsoids of refractive index $n_{2}$, each surrounded by a thin shell $n_{3}$, and immersed in a medium $n_{1}$. A suitable procedure for allowing for this structure may be devised by considering the behaviour of a sphere rather than an ellipsoid, so as to make the electrostatic problem more tractable. Since in the range of values of $n_{1}$ covered in Perutz's measurements $\left(n_{2}-n_{3}\right)$ is considerably greater than $\left(n_{1}-n_{3}\right)$, the major dielectric discontinuity occurs at the protein boundary rather than the watersalt solution boundary. It was therefore decided to treat the composite system as equivalent to a simple system, consisting of a protein molecule of the same size as the true molecule, but with a fictitious refractive index $n_{2}^{\prime}$, in a medium $n_{1}$; the value of $n_{2}^{\prime}$ was determined by direct calculation so that the simple system had the same dipole moment in a given external field as the composite system. It was found that $n_{2}^{\prime}$ decreases very nearly linearly with increase of $n_{1}$, the two becoming identical at that value of $n_{1}$ for which the composite ellipsoid should theoretically cause no nett deviation of the lines of force. If $n_{2}$ is taken as $1 \cdot 60, n_{3}$ as $1 \cdot 33$, and the bound water is taken to have a volume $36 \%$ of that of the protein molecule, the following table of values of $n_{2}^{\prime}$ is found:

$\begin{array}{ll}n_{1} & n_{2}^{\prime} \\ 1 \cdot 33 & 1 \cdot 60 \\ 1 \cdot 38 & 1 \cdot 58 \\ 1.43 & 1 \cdot 56 \\ 1.48 & 1 \cdot 54 \\ 1.526 & 1.526\end{array}$

These values of $n_{2}^{\prime}$ have been used instead of $n_{2}$ in equation (6) in calculating the expected change of birefringence with $n_{1}, f$ being kept constant throughout. It is worth noting that the value 1.526 at which $n_{1}=n_{2}^{\prime}$ is very close to $\bar{n}$, the average value of $n$ in the hydrated protein molecule, 1.529 , so that the calculation of $n_{2}^{\prime}$ may be reduced to a very simple rule:

$$
n_{2}^{\prime} \fallingdotseq \bar{n}+\frac{\left(n_{2}-\bar{n}\right)\left(\bar{n}-n_{1}\right)}{\bar{n}-n_{3}} .
$$

\section{References}

AdaIR, G. S. \& AdaIR, M. E. (1934). Biochem. J. 28, 1230.

BragG, W. L. \& Perdtz, M. F. (1952a). Acta Cryst. 5, 277.

BragG, W. L. \& Perdtz, M. F. (1952b). Acta Cryst. 5, 323.

Perutz, M. F. (1953). Acta Cryst. 6, 859.

RAYLeigh, Lord (1892). Phil.Mag. (6), 34, 481.

Stoner, E. C. (1945). Phil. Mag. (7), 36, 803.

Stratton, J. A. (1941). Electromagnetic Theory, p. 213. New York: McGraw-Hill.

WIENER, O. (1912). Abh. sächs. Ges. (Akad.) Wiss. 32, 509. 